Documentation et bibliothèques

DOCUMENTATION BIBLIOTHEQUES

Une vision technicienne de la bibliothéconomie

A "technical” vision of library science Una visión “técnica” de la biblioteconomía

\title{
Gérard Mercure
}

Volume 26, numéro 4, décembre 1980

URI : https://id.erudit.org/iderudit/1054215ar

DOI : https://doi.org/10.7202/1054215ar

Aller au sommaire du numéro

Éditeur(s)

Association pour l'avancement des sciences et des techniques de la documentation (ASTED)

ISSN

0315-2340 (imprimé)

2291-8949 (numérique)

Découvrir la revue

Citer cet article

Mercure, G. (1980). Une vision technicienne de la bibliothéconomie.

Documentation et bibliothèques, 26(4), 183-196.

https://doi.org/10.7202/1054215ar
Résumé de l'article

Le bibliothécaire applique dans l'exercice de sa profession des techniques qui lui sont propres ou qui sont empruntées à d'autres secteurs d'activité. L'auteur élabore une pensée technicienne qui est en mesure d'unifier ces techniques et d'en éclairer le développement et la pratique. À ces techniques correspond un ensemble d'instruments allant du coupe-papier à l'ordinateur; le présent article étudie leur rôle et leur insertion dans cette technologie des bibliothèques.
Tous droits réservés @ $\subseteq$ Association pour l'avancement des sciences et des techniques de la documentation (ASTED), 1980
Ce document est protégé par la loi sur le droit d'auteur. L'utilisation des services d'Érudit (y compris la reproduction) est assujettie à sa politique d'utilisation que vous pouvez consulter en ligne.

https://apropos.erudit.org/fr/usagers/politique-dutilisation/ 


\title{
Une vision technicienne de la bibliothéconomie
}

\author{
Gérard Mercure \\ Université du Québec à Rimouski
}

Le bibliothécaire applique dans l'exercice de sa profession des techniques qui lui sont propres ou qui sont empruntées à d'autres secteurs d'activité. L'auteur élabore une pensée technicienne qui est en mesure d'unifier ces techniques et d'en éclairer le développement et la pratique. À ces techniques correspond un ensemble d'instruments allant du coupe-papier à l'ordinateur; le présent article étudie leur rôle et leur insertion dans cette technologie des bibliothèques.

\begin{abstract}
A "technical" vision of library science
In the exercise of their profession, librarians use techniques unique to their domain or borrowed from other sectors. The author develops a "technical" approach which is able to unify these techniques, shedding light on their development and application. A group of tools is associated with these techniques, ranging from the paper knife to the computer; the article analyzes the role and presence of these tools in the library technology.
\end{abstract}

\section{Una visión "técnica" de la biblioteconomía}

En el ejercicio de su profesión, el bibliotecario utiliza sus técnicas propias y técnicas que proceden de otros sectores. El autor elabora un pensamiento "técnico" que puede unificar estas técnicas y aclarar su desarrollo y práctica. A estas técnicas corresponde una serie de instrumentos, que incluye tanto el cortapapel como la computadora; el autor estudia en este artículo el papel y la presencia de estos instrumentos en la tecnología de las bibliotecas.

Si la bibliothéconomie n'a pas comme l'astronomie de fascinantes énigmes, elle possède néanmoins quelques "trous noirs" intéressants à scruter. Cet article est né de la curiosité de découvrir ce que peut cacher l'expression "technologie des bibliothèques". Aurons-nous la veine de ce bibliothécaire-astronome japonais, Kuwano, qui en est à la découverte de sa troisième étoile? ${ }^{1}$ A tout événement, nous vous livrons le résultat de nos observations.

Ce terme pourtant employé assez fréquemment dans la littérature professionnelle n'apparaît en vedette ni dans les encyclopédies spé. cialisées ni dans les grands index de la bibliothéconomie. Ou nous sommes sur une fausse piste, ou nous pointons notre télescope sur une

1. "Librarian sees stars", American Libraries, vol. 6 no. 10 (November 1975), 590. 
trop vaste nébuleuse. Serait-ce l'équivalent de "library sciences" ou de "bibliothéconomie"? Pourtant, dans un article du Bulletin de I'Unesco à l'intention des bibliothèques, on retrouve l'expression "library technology", que le traducteur a rendu par "technologie des bibliothèques"'!2

Une consultation de l'Encyclopédie Universalis fera comprendre la raison de notre méprise. La confusion est en partie d'ordre terminologique. Le terme "technologie" se voit constamment substitué à "technique", parce que "plus noble, plus chargé de science, plus avancé que le substantif qu'il a supplanté" 3 . Nous sommes au moins fixés! Pour une fois, ce n'est pas l'origine ancienne de notre profession qui nous prive d'un vocabulaire d'allure scientifique mais la sobriété de la langue française. D'où la nécessité d'employer le mot technologie avec circonspection et de bien le distinguer des autres termes qui gravitent autour de lui: technique, procédé, art, etc.

\section{Un retour dans la nuit des temps}

Une exploration plus poussée du vocabulaire nous entraîne aussi loin dans l'univers que celle du "dévoilement", c'est-à-dire de la vérité. Car selon Heidegger:

Jusqu'à l'époque de Platon, le mot "techne" est toujours associé au mot "épisteme". Tous deux sont des noms de la connaissance au sens le plus large. Ils désignent le fait de pouvoir se retrouver en quelque chose, de s'y reconnaître. ${ }^{4}$

Dès l'Antiquité cependant le partage est fait entre "logos" et "praxis". La technique, liée au corps dont l'outil est le prolongement, devient distante de l'esprit. Le lien étroit unissant les trois réalités, les beaux-arts, le savoir et la technique, a disparu. Ce n'est que plusieurs siècles plus tard qu'une réflexion sur la technique réservera à cette activité humaine une meilleure place dans la culture.

Cette union du rationnel et du pratique ne se fera pas sans peine. Dans les siècles précédant la Renaissance, la philosophie l'emporte sur l'observation du travail de l'artisan. Avec la mécanique de Léonard de Vinci, la conquête du Nouveau Monde, l'industrialisation de l'Europe (les mines et l'artillerie), l'imprimerie de Gutenberg, la technique reprend ses droits. Descartes sera l'un des premiers à se soucier de la formation des artisans de la lunetterie en leur donnant des outils dont les principes de fonctioninement s'appuient sur les données de la géométrie et de la mécanique et en présidant à la fondation des écoles d'arts et métiers par l'énoncé des principes qui les régiront.

Au cours du siècle dernier, la science aura encore tendance à dominer la technique; ce qui fait dire à Herbert Simon:

Les écoles d'ingénieurs sont devenues des écoles de physique

2. Gladys T. Piez, "L'étude de la technologie des bibliothèques: une entreprise de portée mondiale"', Bulletin de l'Unesco à l'intention des bibliothèques, vol. 19, no 5 (septembre - octobre 1965), 249-256.

3. "Technologie", in Encyclopedia Universalis, vol. 15, Paris, $1968=1975$, p. 820-823.

4. Martin Heidegger, "La question technique", in Essais et conférences, Paris, Gallimard, 1958, p. 18. 
et de mathématiques; les écoles de médecine sont devenues des écoles de biologie; les écoles de gestion sont devenues des écoles de mathématiques finies... Ce mouvement vers les sciences naturelles, s'éloignant des sciences de l'artificiel, s'est effectué plus avant et plus rapidement en ingénierie, en gestion et en médecine que dans les autres domaines professionnels que j'ai mentionnés, bien qu'il n'ait pas été absent des écoles de droit ou de journalisme, ni même des écoles de documentalistes. ${ }^{5}$

\section{Les satellites de la bibliothéconomie}

Malgré ses prétentions scientifiques, la bibliothéconomie est fondée davantage sur l'action que sur la motivation au savoir. Les lois qui la gouvernent sont peu nombreuses mais les règles qui en guidení la pratique sont multiples.

La bibliothéconomie s'appuie sur quelques principes généraux qui lui sont propres, telles les cinq lois de la bibliothéconomie formulées par Ranganathan et la bibliologie ou science générale du livre que le Librarians' Glossary assimile à la bibliographie analytique, descriptive ou historique 6 .

Au nombre des sciences connexes figurent la théorie de l'information et la documentation que Jesse $\mathrm{H}$. Shera qualifie respectivement de "bibliothéconomie de type non traditionnel" et de "bibliothéconomie à l'octave supérieure"' " Quant à la science de l'information, cette science des propriétés du comportement de l'information, elle serait, selon Shera, la base théorique de la pratique de la bibliothéconomie. La bibliothéconomie s'inspire également de disciplines plus générales dont la philosophie, la psychologie, la sociologie, et utilise leurs méthodes de recherche.

Les techniques y sont représentées en bien plus grand nombre. Outre les procédés traditionnels propres aux bibliothèques pour leurs opérations, il y a nombre d'emprunts à des techniques débordant largement le champ propre de l'organisation de la documentation.

Sans l'imprimerie, point de bibliothèques modernes. Les techniques reprographiques prolongent l'activité d'édition par la diffusion du document au moyen du microfilm et de la photocopie. À cause de ses affinités avec le traitement des textes et les techniques de bureau, la bibliothéconomie puise largement dans leurs outillages et leurs fournitures. Quant aux techniques nées de l'emploi de l'ordinateur, elles sont maintenant synonymes de mécanisation des bibliothèques. Les techniques des communications permettent de nouveaux modes de fonctionnement et d'échange, le regroupement en réseaux, le recours possible à la télématique et à la télédocumentation.

5. Herbert A. Simon, La science des systèmes, science de l'artificiel, Paris, Éditions de l'Épi, 1974, p. 74.

6. Leonard M. Harrod, The Librarians' Glossary, 3d. ed., London, André Deutsch, 1971, p. 80.

7. Jesse H. Shera, "Bibliothéconomie, documentation et science de l'information", Bulletin de l'Unesco à l'intention des bibliothèques, vol. 22, no 2 (mars - avril 1968), $62-69$. 
Les techniques audiovisuelles ont, elle aussi, modifié profondément l'organisation des bibliothèques par la présence de média documentaires autres que l'imprimé. La technologie éducative. notamment pour les bibliothèques d'enseignement, apporte aussi une connaissance renouvelée du phénomène de l'apprentissage et de l'enseignement individualisé.

À cette rapide énumération, on peut ajouter les techniques de construction et d'aménagement. Le monde des bibliothèques a toujours été attentif au progrès dans ce domaine de l'architecture. II a créé un matériel de rangement qui lui est propre et favorisé une approche modulaire du mobilier avant qu'elle ne soit généralisée dans l'ameublement de bureau.

Une technique étant par définition un ensemble de procédés au service d'un art ou d'une science, il est fréquent de retrouver plusieurs procédés au sein d'une même technique et plusieurs techniques au service d'un même art. Ainsi, le traitement des textes associe à la composition typographique la mémoire graphique de la photographie, l'affichage visuel de la télévision et les données manipulables de l'informatique. Les techniques de la reprographie se retrouvent à la fois dans les techniques de bureau et dans les techniques documentaires. En outre, une technique peut aussi jouer plus d'un rôle dans l'activité humaine; ainsi les techniques audiovisuelles peuvent servir à la vente, à l'enseignement ou aux communications. Cette souplesse de la technique vient de sa nature même. Elle traite du particulier, mais c'est par la synthèse qu'elle domestique le réel.

\section{De nouvelles lois pour un vieil univers?}

N'aurions-nous pas négligé de considérer la bibliothéconomie comme un ensemble de techniques, trop préoccupés que nous étions de puiser notre philosophie et notre culture dans les sciences humaines? II aurait été tout aussi profitable de chercher également notre inspiration du côté de la technologie et de nous laisser guider dans la recherche d'actions satisfaisantes par sa logique impérative plutôt que de chercher une vérité formelle que la technique ne peut démontrer. C'est ce que l'automatisation et l'approche systémique sont en train de nous apprendre.

De formation, nous sommes davantage des philosophes que des ingénieurs. Nous devrions nous transformer en penseurs de la technique pour dégager une technologie de nos propres activités. Les ouvrages sur les rapports de la science et de la technique ne manquent pas. Nombre de penseurs se sont attardés à ce problème: Heidegger, Simon, Auzias, Simondon, Russo, Gille. Étant de langue française, nous sommes favorisés: les philosophes et technologues français ont davantage réfléchi sur les rapports entre la science et la technique que ne l'ont fait leurs collègues anglo-saxons plus intéressés à l'histoire qu'à la philosophie.

Ces théoriciens insistent sur le fait que la technique est porteuse d'un savoir qui n'est pas la simple application d'une science qui lui serait supérieure. Elle vise à un savoir utile et efficace. C'est une culture en acte qui se développe au sein de la réalité concrète plutôt que par 
la spéculation. Toutefois, la différence entre le savoir de la science et le savoir de la technique est de moins en moins tranchée. La technique moderne fournit à la science même les instruments de laboratoire pour la maîtrise de son savoir. La science qui est contemplation dans sa fin est aussi action dans sa démarche. Plutôt que de partager cette action en deux histoires, l'une pour les sciences et l'autre pour les techniques, Russo esquisse une histoire "de la maîtrise de l'homme sur les "objets"." ou l'histoire "de l'enrichissement de la nature par des réalités artificielles" 8

Pour Simondon, "la technicité tend à résider dans les ensembles; elle peut alors devenir un fondement de la culture à laquelle elle apportera un pouvoir d'unité et de stabilité, en la rendant adéquate à la réalité qu'elle exprime et qu'elle règle"' 9

C'est grâce à cette science de la conception, cette "technologie", que se fera l'union du rationnel et du pratique. Herbert Simon ajoute:

Les écoles professionnelles pourront assumer effectivement leurs responsabilités professionnelles dans la mesure où elles pourront découvrir une science de la conception, un corps de doctrine sur les processus de la conception qui soit solide intellectuellement, analytique, partiellement formalisable, partiellement empirique ${ }^{10}$

Une bibliothéconomie trop cérébrale ne saurait que discourir, une bibliothéconomie trop pragmatique n'agirait qu'à courte vue. Sans un langage partiellement commun, elles ne pourraient communiquer. L'approche technologique leur permet de faire appel à une même approche raisonnée, et leur confère un même objectif d'unification de la réflexion et de l'agir. À en juger par ce qui s'est écrit jusqu'à ce jour sur la mécanisation des bibliothèques, on peut affirmer que l'on en a traité beaucoup plus à la forme active que réfléchie.

Cette approche technologique de la bibliothéconomie pourrait être une vision originale du bibliothécaire qui voit et nomme ce nouvel ensemble de procédés et d'outils, en fait le discours et l'intègre aux activités de sa bibliothèque selon ses besoins et son génie, plutôt que de l'observer le plus souvent du service de l'informatique. II pourrait ainsi la faire sienne plutôt que d'y recourir par contrats de services.

On peut alléguer que la technique n'est pas le fait du bibliothécaire, mais de l'ingénieur. Bien que la pensée technicienne soit d'abord identifiée à l'art de ce dernier, elle appartient également au médecin, au politicien, et à tout agent de l'industrie humaine.

Tout comme la technologie apprend à l'ingénieur à organiser logiquement la machine qu'il veut construire par une utilisation correcte des lois de la physique, de la chimie, avec le souci de la fiabilité, une connaissance des moyens pratiques pour y arriver, un souci de rentabilité, la bibliothéconomie doit appliquer la même rigueur de pensée à

8. François Russo, "Science et technique", in Histoire des techniques, Paris, Gallimard, 1978, p. 1111-1145.

9. Gilbert Simondon, Du mode d'existence des objets techniques, Paris, AubierMontaigne, 1969, p. 16.

10. Herbert A. Simon, "La science... .", p. 76. 
la conception et à la realisation d'un système documentaire. Bien que non réductible à une machine, la bibliothèque n'en possède pas moins ses organes de commande, de transmission et de contrôle, ses techniques, ses outils et ses procédés.

\section{Pour une vision renouvelée du monde de la documentation}

La bibliothéconomie, vue sous ce jour, plus que l'étude de la documentation sous toutes ses formes ou le fonctionnement des bibliothèques, vise la réponse à une problématique de la conservation et de la diffusion de l'information documentaire qui tient compte de la réalité politique, économique et sociale. Depuis quelques années, une réponse diversifiée a donné lieu à plusieurs modèles d'information et également à des industries parallèles. II n'est pas certain que la bibliothèque actuelle soit un outil adapté au nouvel environnement. À quels critères devrait-elle répondre: à la rentabilité, à la rapidité, à la fiabilité, à l'accessibilité? Ce sont des interrogations familières à l'ingénieur que doit aussi se poser le bibliothécaire.

D'autres modèles de diffusion peuvent être envisagés grâce aux possibilités nouvelles de la technique. Le modèle actuel, issu de l'imprimerie, a favorisé jusqu'à ce jour le transfert de l'information; il repose sur le fait qu'en multipliant mécaniquement les exemplaires du livre - cette machine à lire - il est possible de rejoindre un grand nombre de lecteurs. Un nouveau modèle, qui tire sa puissance de la reprographie, l'électronique et les télécommunications, mise sur un ensemble de moyens plutôt que sur un seul, comme c'était le cas avec l'imprimerie: choix du support, choix de la ligne de communication et choix du mode de distribution.

Un tel modèle risque de modifier considérablement le rôle de la bibliothèque: rationalisation des fonds rendue plus facile par l'ubiquité et l'instantanéité des communications électroniques, gestion allégée par la décentralisation des catalogues et la simplification du prêt, le document étant libéré de son support d'origine, réduction des installations par la miniaturisation et l'avènement du service à domicile. C'est encore pour le moment la bibliothèque du meilleur des mondes, mais on peut penser que les grands bouleversements de la bibliothéconomie viendront plutôt des transformations apportées par la technique que par une découverte scientifique de quelque loi de la relativité régissant le monde de la documentation.

\section{Une approche épistémologique des techniques du bibliothécaire}

Pour que l'action ne devance pas encore une fois la pensée, arrêtons-nous, dans notre démarche, à trois aspects globaux des techniques du bibliothécaire: un rapide inventaire de ses outils, accompagné d'un essai de classement, un aperçu historique de la mécanisation des bibliothèques et une brève réflexion sur la nature de l'instrument réservé aux fins de sa profession.

Le bibliothécaire est sans doute l'un des professionnels les mieux outillés dans son domaine. Il peut compter sur un nombre impression- 
nant de répertoires, d'index, d'annuaires dans le seul domaine de la bibliothéconomie, ouvrages qu'il appelle d'ailleurs ses "instruments bibliographiques".

Mais il en est d'autres dont il ne dépend pas moins dans l'exercice de ses fonctions. À l'exclusion de l'ordinateur, ces autres instruments que sont les machines qui l'assistent dans l'exécution de son travail quotidien reçoivent peu d'attention de sa part. Ainsi, il ne consultera qu'à l'occasion des guides comme Library Technology Reports et Micrographics Equipment Review pour l'achat de son équipement.

En faisant l'inventaire des outillages utilisés dans une bibliothèque, on sera surpris de l'importance du matériel investi aux fins du traitement de la documentation et du service à la clientèle.

II y a d'abord "les ustensiles" que sont les petits outils et les fournitures allant des dateurs et estampes au massicot, en passant par la "crème à compter les pages". Viennent ensuite les appareils, machines à écrire, à photocopier et les appareils plus spécialisés de reliure, de microfilm, de prêt et de contrôle anti-vol. De plus, n'oublions pas les équipements lourds que sont les ordinateurs avec leurs terminaux périphériques et leurs logiciels. Enfin, il faut mentionner les fichiers qui font partie des meubles à caractère fonctionnel, comme l'établi pour le menuisier.

Les voici regroupés selon leurs techniques d'origine:

- l'édition et les arts plastiques

- la reprographie

les techniques de bureau

les techniques de l'informatique le livre

les appareils et fournitures de reliure le massicot

les imprimeries manuelles

la trousse de réparation matérielle

le babillard, l'affiche, le poster

la photocopie, le microfilm

le photocopieur

le microcopieur

les lecteurs de microfilm

les crayons et stylos

la machine à écrire

le dictaphone

la machine à calculer

les partitions mobiles

le classeur

les matériels de rangement

les formulaires

la liste de sortie

le terminal

l'ordinateur

les périphériques

les langages et programmes

les télécommunications le télex

le téléphone

le téléviseur 
le télécopieur

les lignes de données

- les techniques audiovisuelles

- les techniques de construction photographie, diapositive, film, disque, bande, etc.

les matériels audiovisuels: magnétophones, magnétoscopes, caméras, appareils de photo, projecteurs, épiscopes, etc.

\author{
les normes \\ les matériaux \\ le mobilier: \\ isoloirs, fichiers, présentoirs, comptoirs, \\ chariots \\ le rayonnage
}

À ces outils tangibles, il faut ajouter les techniques de gestion, les techniques éducatives et de bureau qui sont davantage des procédés que des outils, mais d'une utilité toute aussi quotidienne.

Voyons ce que peut nous révéler leur classement selon leur fonction principale, analogue, ou propre à un appareil destiné au traitement d'un signal ou d'une information.

Les appareils scientifiques et techniques sont identifiés par leur fonction principale: I'observation, la mesure, le contrôle, le calcul, la communication ou l'enregistrement. Le bibliothécaire ne possède pratiquement pas d'instruments d'observation ou de constatation attentive d'un phénomène; il en possède peu pour la mesure, quelquesuns pour le contrôle de ses opérations et plusieurs pour la communication et l'enregistrement de l'information.

On peut également les regrouper selon les fonctions analogues aux sens qu'ils amplifient ou remplacent: la vue, le toucher, l'odorat, le maintien de l'équilibre et l'appréciation des distances, ou aux facultés qu'ils assistent. Le regroupement qui leur convient le mieux n'est pas celui par les sens mais par les facultés: assimiler, raisonner, mémoriser, comparer, compter. Est-ce dire que le bibliothécaire cherche d'abord par ses outils le prolongement de son esprit plus que de sa main?

On peut encore les classer selon les fonctions propres au traitement d'un signal ou d'une donnée, - il y en a plus d'une vingtaine dont l'excitation, la modulation, la détection, la comparaison, l'amplification, etc. l'analyse, le contrôle et le calcul. On peut dresser difficilement la liste des appareils du bibliothécaire en regard de ces catégories. La plupart n'ont pas de rapport direct avec l'une ou l'autre de ces fonctions sinon l'ordinateur avec ses périphériques qui occupe presque tout le champ.

Ce petit exercice de classement, familier au bibliothécaire, montre la prédilection de la bibliothéconomie pour les fonctions d'enregistrement et de transcription. Contrairement à d'autres techniques, la bibliothéconomie a fortement recours à l'écrit; elle a peu de tours de mains qui ne s'écrivent pas. L'écrit lui sert de mémoire pour consigner ses modes d'opération. Le livre est à la fois son objet, son produit transformé et son outil. Elle fait peu appel à la mesure et au calcul, qui 
n'est pas son fort, mais elle lorgne vers les mathématiques grâce à la simulation, nouvel outil scientifique qui lui permettra d'échapper à l'empirisme d'une bibliothéconomie artisanale.

\section{Les trois âges de la mécanisation des bibliothèques}

Les bibliothèques sont passées en moins d'un siècle de l'opération manuelle à l'opération automatisée.

\section{L’âge mécanique}

Metcalf voit dans l'étude de Michel-Ange sur les stalles de la Bibliothèque Laurentienne (1525) de Florence et dans la roue-à-livres de Ramelli (1588) qui sert de frontispice au classique de la construction de bibliothèques de cet auteur les lointains débuts de la bibliothèque moderne ${ }^{11}$. Sans doute trouverait-on en cherchant un peu d'autres étincelles de mécanisation, telle cette curiosité attribuée à Benjamin Franklin (1706 - 1790), consistant en un "bras mécanique grâce auquel il pouvait atteindre et faire se poser près de lui les livres de sa bibliothèque, - si haut perchés qu'ils fussent" ${ }^{12}$. Le premier code de catalogage, qui fut de bien plus grande conséquence, ainsi que la fiche de catalogue, inspirée de la carte à jouer, seraient apparus pendant la Révolution française.

La mécanisation des bibliothèques commence vraiment avec Dewey, qui cherche de nouveaux dispositifs et de nouvelles méthodes susceptibles d'aider le bibliothécaire dans son travail. Cet effort prend d'abord la forme d'un comité de l'American Library Association, le Supplies Committee (1877 - 1879) dont l'objectif est d'évaluer les fournitures et les équipements de bibliothèques, pour aboutir à la formation d'une entreprise commerciale, le Library Bureau.

La machine à écrire est le symbole de cette période. Introduite par Dewey en 1885 dans les bibliothèques, elle est l'objet d'une lente adoption par les services techniques. La calligraphie subsistera jusqu'à la Deuxième Guerre malgré les avantages de la dactylographie. Comme la machine à coudre dans l'industrie du vêtement, le dactylographe va permettre de faire plus vite, mieux et avec moins d'effort ce qui se fait déjà à la main.

\section{L’âge automatique}

Dewey n'est pas le seul "bricoleur" de la bibliothéconomie. C'est à la suggestion du directeur de la bibliothèque publique de New York que Hollerith utilise pour la première fois en 1880 la carte perforée pour la compilation d'un recensement. Cette carte trouvera beaucoup plus tard, en 1936, son application dans une bibliothèque du Texas pour le contrôle du prêt. Autre technique ancienne, le microfilm sera introduit dans les bibliothèques de façon artisanale au cours des années 1930, à partir de bricolages de Leica 35 et de ciné-caméras modifiées.

11. Keys D. Metcalf, Planning Academic and Research Library Buildings, New York, McGraw-Hill, 1965, p. 14.

12. Pierre Ducassé, Histoire des techniques, 7e éd. mise à jour, Paris, P.U.F., 1968, p. 75. (Que sais-je, no 126). 
Il faudra la rescousse d'une nouvelle entreprise commerciale, Recordak, pour qu'apparaisse en 1935 une micro-édition rétrospective d'un journal, le New York Times. Le premier sélecteur optique de microfilm fut réalisé par un autre bibliothécaire, Ralf $R$. Shaw, au cours des années 1940.

Malgré ces innovations spectaculaires, le bibliothécaire est encore aux prises avec des problèmes d'étiquettes qui ne collent pas. À l'ère des grandes rotatives, il ne peut reproduire décemment cinq fiches sans en gâcher cinq autres. Chacun y va de sa recette et de son procédé maison. II existait, tout récemment encore, au moins 22 façons de reproduire des fiches! Grâce aux progrès de la reprographie, le bibliothécaire règle enfin ce grave problème domestique.

Au musée des automates de cette époque, outre la Xerox 914, il faudrait exposer les cartes IBM et les trieuses mécaniques, qui laissèrent entrevoir aux bibliothécaires des perspectives nouvelles de traitement et de diffusion de l'information.

Le développement de l'outillage spécialisé est jusqu'alors laissé à l'ingéniosité des gens et à l'initiative de l'industrie. Des organismes de recherche prennent le relai et participent à la mise au point du matériel avec des moyens financiers importants et des modes de gestion scientifiques. Sont tout à tour créés le Council on Library Resources (1956) et le Library Technology Program (1957) aux États-Unis, le National Reprographic Center for Documentation (1967) en Grande-Bretagne, pour ne nommer que les plus connus. Malgré les sommes investies et le sérieux de la recherche de ces organismes, les percées ne sont pas très importantes: quelques innovations, des évaluations en laboratoire de produits sur le marché et des normes élaborées à l'intention de l'industrie. La mise en place de grands systèmes informatisés et la création de réseaux nationaux de documentation absorberont bientôt l'attention des centres de recherche sur les bibliothèques.

\section{L’âge automatisé}

Les bibliothèques passent à l'âge de l'automatisation sans même la transition du "voir aussi" 13. Celle-ci absorbe à ce point les efforts de réflexion qu'elle distrait le monde de la documentation des autres aspects du développement technique des bibliothèques. La première liste automatisée de périodiques est produite en 1962 à l'Université de Californie. MEDLARS est mis en chantier en 1961 et le MARC en 1965. L'Université de Toronto et I'Université Laval joignent le club informatique en 1963.

Les journalistes présentent à grand fracas les innovations. Les informaticiens et analystes, à partir du techniquement faisable, y vont de leurs prédictions, et les administrateurs de bibliothèques un peu crédules instituent des programmes de développement. II y a surenchère et aussi désillusion. II n'y a pas toujours cet équilibre nécessaire entre les innovateurs enthousiastes, les praticiens inquiets et les critiques sévères. À la période euphorique des années 1960 succède

13. Virginia Oliver, Non-computer Mechanized Support Equipment for Libraries, Washington, D.C., Department of the Army, 1971, 45 p. (TISA Project, 35). 
une période de prudence attentive. La bibliothèque futuriste des années 2000, maintenant trop proche, est reportée à l'an 2030.

En technique, la source ne jaillit pas toujours où on l'a creusée. Qui aurait pu deviner, il y a 20 ans, que la meilleure façon de produire des fiches, c'était de les faire disparaître? Selon De Gennaro, bien des modèles élaborés en 1968 ne sont plus viables ${ }^{14}$. Qui pouvait, par exemple, prévoir le virage de l'automatisation du côté du développement des réseaux, des "mini" et des "micro" et des terminaux intelligents?

L'histoire de la mécanisation des bibliothèques est à l'image de I'histoire des techniques, qui se développe par à-coups et de façon interdépendante. Plus une technique peut bénéficier de l'éclairage de plusieurs sciences et de l'appui de plusieurs autres techniques, plus elle est féconde.

On ne pourrait plus penser aujourd'hui à une technique documentaire exclusivement centrée sur l'usage du microfilm comme ce fut le cas de l'école des documentalistes américains des années 1930. La technique du microfilm elle-même a dû s'allier à l'informatique pour demeurer dans la course. L'histoire démontre qu'il est dangereux pour une technique de se livrer pieds et mains liés à la domination d'une seule science ou d'une seule technique. II y aura à plus ou moins longue échéance blocage, assimilation ou élimination.

À tout prendre, la domination de l'ordinateur est peut-être moins dangereuse qu'on pourrait le penser. II est au carrefour de nombreuses techniques, notamment des télécommunications et de la reprographie qu'il va intégrer de façon plus étroite encore aux techniques des bibliothèques.

Une technologie éclairée permettra d'éviter le piège de l'accaparement, car elle sera un regard critique de l'extérieur. Plus permanente que la technique, elle est le discours qui ne s'efface pas. Elle permettra de discerner ce qui est sédiment de techniques antérieures et courant nouveau par lequel la bibliothéconomie peut se laisser porter.

\section{L'instrument du bibliothécaire: ni scientifique ni industriel}

La bibliothèque n'est ni un laboratoire ni une usine, mais tient des deux à la fois. La bibliothéconomie est une science sans microscope ni éprouvettes. Elle puise ses instruments d'observation et de mesure dans des techniques de recherche empruntées aux sciences humaines: études statistiques, historiques et sociologiques. Seul l'ordinateur peut revendiquer le titre d'instrument d'observation et de recherche du monde de la documentation par sa capacité de simulation d'un phénomène et d'analyse des données. C'est sans doute parce qu'il est à la fois un puissant instrument de recherche et de production qu'il fascine à ce point la profession.

14. Richard De Gennaro, "Library automation: changing patterns and new directions". Library Journal, vol. 101, no. 1 (January 1976), 175-183. 
L'introduction d'un nouvel instrument dans une bibliothèque est plutôt liée à l'amélioration d'un rendement et à la qualité d'un service. II est acquis pour ses caractéristiques industrielles car l'appareil industriel produit, transforme, décuple le rendement. Mais c'est surtout comme appareil spécialisé, destiné aux fins d'une profession, qu'il peut être considéré. La technologie médicale, par exemple, a donné naissance à une très grande variété d'instruments. Dans certains secteurs d'activités, s'ajoute à la mesure et l'observation le souci de la production, sans toutefois que cet aspect soit aussi marqué que pour l'outillage destiné à la fabrication industrielle. C'est le cas des appareils identifiés à une profession, tels le théodolite de l'arpenteurgéomètre et le stéréoscope à miroirs du géographe. Le bibliothécaire n'a pas à vrai dire d'appareil qui caractérise son activité, sinon sa bibliothèque qui est en fait son instrument professionnel.

Dans la dynamique de développement des instruments spécialisés, deux facteurs favorisent le progrès: la présence d'une industrie déjà identifiée à la fabrication d'une famille d'équipements et surtout la présence d'un marché suffisamment vaste. II suffit de consulter les revues spécialisées de techniques médicales, maritimes, pétrolières pour identifier les fabricants les plus importants. II existe même des revues traitant exclusivement des instruments de physique ou des équipements industriels. Les bibliothèques n'ont pas de tradition dans ce secteur d'activité et les compagnies spécialisées dans l'équipement lourd de bibliothèques sont rarissimes. Les fournisseurs identifiés au secteur des bibliothèques - plus nombreux en Amérique du Nord qu'en Europe - se concentrent dans la fabrication et la distribution d'un outillage simple, les plus importants étant les systèmes de prêt et de contrôle automatiques et les équipements audiovisuels. Pour des produits plus complexes tels que les matériels de bureau, de reprographie, d'informatique, il faut faire appel aux multiples fournisseurs identifiés à d'autres secteurs.

La gamme restreinte de produits est à l'image du marché étroit que représentent les bibliothèques. À l'échelle même de la petite entreprise et de la petite série, les 30,000 bibliothèques du continent nord-américain sont à peine suffisantes pour susciter une activité commerciale intéressante. Les fabricants importants consentiront tout au plus à des modifications mineures sur un produit déjà conçu pour un plus large public.

C'est du marché de l'équipement de bureau que vient principalement l'innovation dans l'outillage des bibliothèques. On se dirige d'abord vers l'industrie et le commerce qui sont les premières clientèles. Viennent ensuite l'administration publique et les milieux de l'éducation, s'ils y trouvent leur compte. Souvent, les bibliothèques seront perdantes: pensons au retrait graduel du marché du microfilm $35 \mathrm{~mm}$ au profit du $16 \mathrm{~mm}$ moins adapté à la micro-édition et à la prise de vues des journaux. Elles peuvent aussi jouer gagnant, comme avec les ordinateurs qui se rapprochent du devis particulier imposé par le traitement de la documentation, devis fort différent, paraît-il, de l'ordinateur destiné au traitement de données numériques. Les bibliothèques seront aidées ici par le marché florissant de l'industrie de l'information et par l'accessibilité de l'ordinateur à la petite et moyenne entreprise.

Au cours des années 1960, les bibliothécaires se sont impliqués 
directement, comme nous l'avons vu, dans le développement d'appareils. Depuis, ils ont délaissé cette activité pour jouer surtout un rôle de chien de garde dans l'instauration et la défense de normes de qualité et de castor ingénieux à l'affût de tout ce qui enrichit l'environnement, la dernière adaptation étant celle du SOM aux exigences particulières de la bibliographie. Ils ont compris que leur rôle innovateur est limité par la nature même du produit. L'appareil du bibliothécaire n'a ni la noblesse de l'instrument scientifique, ni la puissance de la machine industrielle, moins encore le poids économique de l'appareil de grande consommation.

\section{Les retombées pour le bibliothécaire et le technicien}

En reconnaissant la valeur culturelle de la technique, le bibliothécaire aura plus de considération pour la "plomberie" de sa profession. Son intérêt pour l'outillage dépassera la visite annuelle à l'exposition des matériels dans le cadre des congrès. Sa familiarité avec la documentation sur le sujet ira au-delà de la consultation des guides du consommateur au moment de l'achat d'un terminal. Les écoles de bibliothéconomie placeront au même rang les fournisseurs de livres et les fournisseurs de techniques et traiteront d'organisation et de conditions du marché ainsi que des répercussions de la production sur les techniques documentaires. Les périodiques spécialisés feront la revue des brevets et inventions touchant le secteur, tout comme elles le font pour le reste de la documentation professionnelle. Les organismes de recherche consacreront une part importante de leurs nobles efforts au développement de systèmes, avec la même motivation intellectuelle qu'ils accordent à la découverte de nouvelles lois à l'appui de leurs théories sur le message documentaire.

Tout en demeurant intéressé à la solution administrative, le bibliothécaire participera de façon plus active et plus gratuite à l'effort d'innovation technologique. La technologie "donne un support à l'intuition" en permettant une exploration méthodique de "tout ce qui est possible pour résoudre un problème" 15. Une forme de créativité est propre à la technique, l'activité d'invention.

L'invention n'est pas seulement une réaction adaptative et défensive; elle est une opération mentale, un fonctionnement mental qui est du même ordre que le savoir scientifique $\ldots{ }^{16}$

C'est moins dans la création d'appareils que dans le développement de systèmes qu'il pourra innover: création de logiciels pour le traitement des documents et l'interface documentation-usager. C'est actuellement le créneau le plus accessible au bibliothécaire et l'une des raisons pour lesquelles, dans son esprit, mécanisation et ordinateur sont si intimement associés à l'innovation dans son secteur.

Son attention étant moins polarisée par l'ordinateur, le bibliothécaire exigera une approche plus unifiée du développement de ses instruments de travail. II trouvera dans une technologie des biblio. thèques un appui rationnel plus rigoureux. L'approche systémique, plus 
qu'une recette pour la mise en oeuvre du projet, deviendra la grammaire de son discours. Tout comme une méthodologie est le guide du travail intellectuel, elle le conseillera dans son action. Le bibliothécaire évitera ainsi des erreurs coûteuses de développement, y compris la plus élémentaire qui est de confondre souvent dans la pratique développement et exploitation.

La mécanisation supprime les opérations répétitives, mais du même coup atténue la perception directe sinon de l'utilité du travail du moins de l'évidence de l'action de l'opérateur. Elle masque le sens artisanal qui valorisait l'habileté du technicien. Remplir un bordereau ou modifier au terminal une description bibliographique n'a plus la même signification immédiate que la préparation d'un jeu de fiches. Par sa capacité d'interprétation du fonctionnement de la machine, le technicien apportera une participation personnelle: son savoir technique prolongera l'activité d'invention. Et même l'entretien de l'équipement, qui est le prolongement de sa construction, en sera facilité, une bonne compréhension prévenant ou détectant les pannes avec plus de perspicacité.

Au cours de la formation du technicien, on devrait lui proposer une réflexion globale sur ses techniques de travail avant qu'il entreprenne l'étude séparée de chacune d'elles.

\section{La bibliothéconomie, une technologie?}

La bibliothéconomie n'est pas à proprement parler une technologie puisqu'elle n'est pas ce discours raisonné de la technique, cette science de l'artificiel. Elle s'en approche dans la mesure où elle regroupe, à l'image de la technologie, un ensemble de connaissances sur les "techniques, outils, appareils, matériaux qui sont utilisés en vue d'une action définie, dans un milieu humain, économique, géographique déterminé et à une époque donnée" 17 . Elle est par extension une technologie de spécialité. C'est donc dans un sens restreint que l'on peut parler de technologie des bibliothèques ou de la documentation, et non sans un certain risque de confusion. Cette technologie particulière devra, pour être reconnue comme telle, élaborer une théorie généralisée et donner lieu à une réflexion, à un discours sur l'ensemble des techniques du bibliothécaire.

Qu'on se rassure donc, le monde des bibliothèques ne risque pas d'être aspiré par une anti-bibliothéconomie, même s'il est envahi par des robots venant d'un univers autre que le sien! Sans s'opposer ni se détruire, science et technologie des bibliothèques forment plutôt une étoile double dans le ciel de la connaissance documentaire. Et comme pour ces doubles étoiles dont on a soupçonné l'existence par le calcul avant de les localiser par la lunette, il nous faudra en poursuivre le repérage. Peut-être un jour, à l'exemple de ce collègue astronome, pourrons-nous demander à notre profession que sa découverte soit homologuée... 